\title{
AN OPERATIONAL DESCRIPTION OF THE COMPACT \\ $\because \quad$ AND TUNABLE LEP SPIN ROTATOR
}

\author{
T. Fieguth \\ Stanford Linear Accelerator Center, Stanford University, Stanford, CA 94309, USA \\ G. Guignard \\ CERN CH-1211, Geneve 23, Switzerland
}

\begin{abstract}
Special configurations of horizontal and vertical bending magnets can rotate the spin vector from the vertical to the longitudinal direction. The compact and tunable LEP spin rotator discovered recently fits neatly into the list of such configurations that generate a group of 24 rotation operators. It is shown that any of these configurations can be achieved with a string of 8 simple dipoles. Results can, in principle, be applied to electron or proton beams.
\end{abstract}

\section{Introduction}

It has been shown ${ }^{1}$ that it is possible to find special configurations of horizontal and vertical bending magnets which will rotate the spin polarization vector from the vertical (or horizontal) direction to the longitudinal direction and at the same time have the following important attributes.

1. The configuration can be inserted into a drift region and will not change the overall direction of the beam.

2. The operation performed on the spin polarization vector is independent of the energy of the beam.

3. The configurations do not induce dispersion in the regions outside of the region of the configuration itself.

In Ref. 1, it was also shown that these configurations and combinations of them could generate a group of 24 operators isomorphic to the group of rotational symmetries of a cube (Octahedral Group).

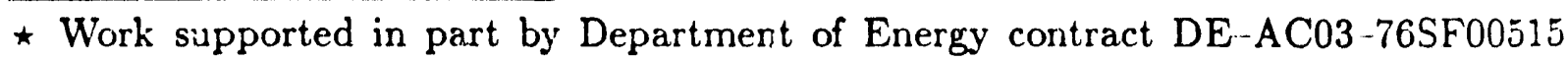
while visiting CERN. 
Recently an investigation ${ }^{2}$ of configurations of this type lead to the discovery of one previously unreported which also is the most economic in that it requires less integrated field than those previously known. When the operation which it performs on the three components of the spin vector was examined it was found that it neatly provided the two operations needed to complete the list of simple magnet configurations which rotate vertical spin into longitudinal spin. This new configuration is called the Compact. L.EP Rotator.

\section{Operation of the Compact LEP Rotator}

To describe the operation of the new Vertical Compact LEP Rotator (VLR) on the components of the spin polarization vector we use the same conventions described in Ref. 1 where $\mathbf{V}\left(+45^{\circ}\right)$ and $\mathbf{H}\left(+90^{\circ}\right)$ denote the orthogonal $(3 \times 3)$ matrix operators representing precession of $+45^{\circ}$ or $+90^{\circ}$ in vertical or horizontal bending magnets. The new VLR configuration of magnets is shown in Fig. 1, where only its action on the vertical component of the initial spin vector is shown. The overall operation of this configuration on all of the components of the spin vector $\mathbf{p}$ is obtained by the sequential application of the operator for each bend magnet.

$$
\mathbf{p}^{\prime}=\mathbf{V}\left(+45^{\circ}\right) \mathbf{H}\left(+90^{\circ}\right) \mathbf{V}\left(-45^{\circ}\right) \mathbf{H}\left(-180^{\circ}\right) \mathbf{V}\left(-45^{\circ}\right) \mathbf{H}\left(+90^{\circ}\right) \mathbf{V}\left(+45^{\circ}\right) \mathbf{p}
$$

We will represent this configuration by the notation, $\operatorname{VLR}\left(+45^{\circ},+90^{\circ}\right)$ and rewrite Eq. (2.1) as

$$
\mathbf{p}^{\prime}=\mathbf{V L R}\left(+45^{\circ},+90^{\circ}\right) \mathbf{p}
$$

which after the matrix calculation becomes,

$$
\mathbf{p}^{\prime}=\left(\begin{array}{ccc}
1 & 0 & 0 \\
0 & 0 & -1 \\
0 & 1 & 0
\end{array}\right)\left(\begin{array}{l}
H \\
V \\
S
\end{array}\right) \quad \text { or } \quad \mathbf{p}^{\prime}=\left(\begin{array}{c}
H \\
-S \\
V
\end{array}\right)
$$

As expected, we note that the vertical component $V$ of the initial vector has now been rotated to become the longitudinal component. The matrix operator is described in Ref. 1 and given an identifying label $\mathbf{E}_{3}$. We could now write

$$
\mathbf{p}^{\prime}=\mathbf{E}_{\mathbf{3}} \mathbf{p}
$$

The operator $\mathbf{E}_{3}$ is but one of 24 operators representing the group of rotational symmetries of a cube. In Ref. 1, all these operators are described in both matrix form and in a second more compact form whereby the eigenvector (rotational axis) is calculated and specified along with a rotational angle about that axis. Thus the operator $\mathbf{E}_{3}$ represents a rotation of $+90^{\circ}$ about the unit vector $+\mathbf{i}$ which is along the horizontal axis. The complete list of all such operators is given in Table I.

By permuting the field directions and recalculating we obtain: 

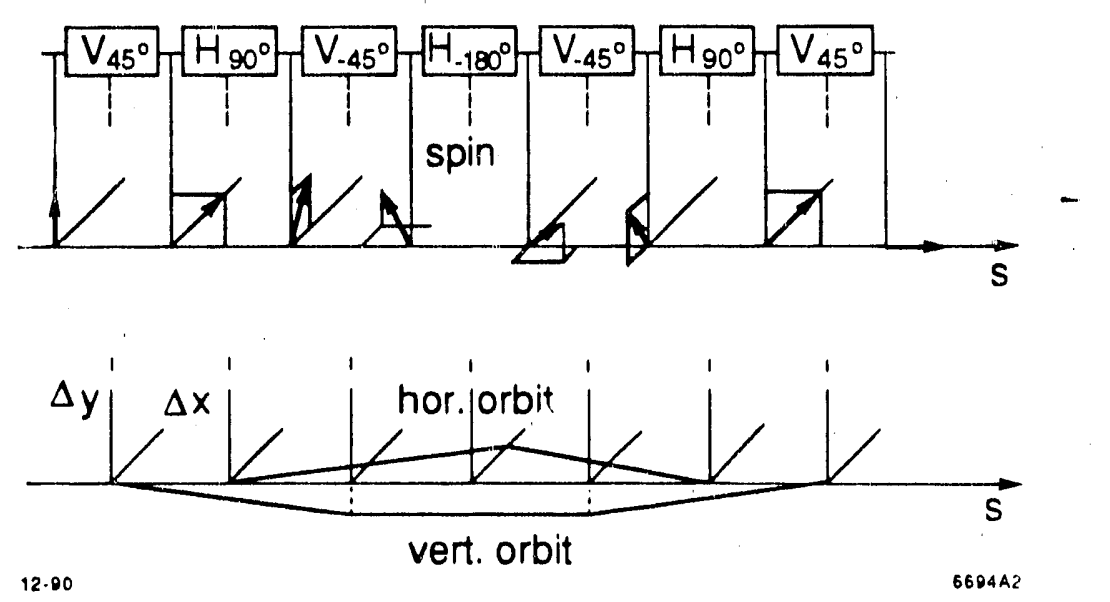

Figure 1: The 7 VLR magnets, orbit and action on vertical spin component.

Table I: Summary of the 24 operators of the Octahedral Group with respective rotation angles and eigenvectors (rotation axes).

\begin{tabular}{|c|c|c|}
\hline $\mathbf{A}_{1}, \mathbf{A}_{1}^{-1}$ & $+120^{\circ},-120^{\circ}$ & $\mathbf{a}_{1}=1 / 3(+\sqrt{3} \mathbf{i}+\sqrt{3} \mathbf{j}+\sqrt{3} \mathbf{k})$ \\
$\mathbf{A}_{2}, \mathbf{A}_{2}^{-1}$ & $+120^{\circ},-120^{\circ}$ & $\mathbf{a}_{2}=1 / 3(+\sqrt{3} \mathbf{i}-\sqrt{3} \mathbf{j}-\sqrt{3} \mathbf{k})$ \\
$\mathbf{A}_{3}, \mathbf{A}_{3}^{-1}$ & $+120^{\circ},-120^{\circ}$ & $\mathbf{a}_{3}=1 / 3(-\sqrt{3} \mathbf{i}+\sqrt{3} \mathbf{j}-\sqrt{3} \mathbf{k})$ \\
$\mathbf{A}_{4}, \mathbf{A}_{4}^{-1}$ & $+120^{\circ},-120^{\circ}$ & $\mathbf{a}_{4}=1 / 3(-\sqrt{3} \mathbf{i}-\sqrt{3} \mathbf{j}+\sqrt{3} \mathbf{k})$ \\
\hline $\mathbf{C}_{1}$ & $180^{\circ}$ & $\mathbf{c}_{1}= \pm \mathbf{k}$ \\
$\mathbf{C}_{2}$ & $180^{\circ}$ & $\mathbf{c}_{2}= \pm \mathbf{i}$ \\
$\mathbf{C}_{3}$ & $180^{\circ}$ & $\mathbf{c}_{3}= \pm \mathbf{j}$ \\
$\mathbf{I}$ & $0^{\circ}$ & \\
\hline $\mathbf{D}_{1}$ & $180^{\circ}$ & $\mathbf{d}_{1}=1 / 2( \pm \sqrt{2} \mathbf{i} \mp \sqrt{2} \mathbf{k})$ \\
$\mathbf{D}_{2}$ & $180^{\circ}$ & $\mathbf{d}_{2}=1 / 2( \pm \sqrt{2} \mathbf{i} \pm \sqrt{2} \mathbf{k})$ \\
$\mathbf{D}_{3}, \mathbf{D}_{3}^{-1}$ & $+90^{\circ},-90^{\circ}$ & $\mathbf{d}_{3}=+\mathbf{j}$ \\
\hline $\mathbf{E}_{1}$ & $180^{\circ}$ & $\mathbf{e}_{1}=1 / 2( \pm \sqrt{2} \mathbf{j} \mp \sqrt{2} \mathbf{k})$ \\
$\mathbf{E}_{2}$ & $180^{\circ}$ & $\mathbf{e}_{2}=1 / 2( \pm \sqrt{2} \mathbf{j} \pm \sqrt{2} \mathbf{k})$ \\
$\mathbf{E}_{3}, \mathbf{E}_{3}^{-1}$ & $+90^{\circ},-90^{\circ}$ & $\mathbf{e}_{3}=+\mathbf{i}$ \\
\hline $\mathbf{S}_{1}$ & $180^{\circ}$ & $\mathbf{s}_{1}=1 / 2( \pm \sqrt{2} \mathbf{i} \pm \sqrt{2} \mathbf{j})$ \\
$\mathbf{S}_{2}$ & $180^{\circ}$ & $\mathbf{s}_{2}=1 / 2( \pm \sqrt{2} \mathbf{i} \mp \sqrt{2} \mathbf{j})$ \\
$\mathbf{S}_{3}, \mathbf{S}_{3}^{-1}$ & $+90^{\circ},-90^{\circ}$ & $\mathbf{s}=+\mathbf{k}$ \\
\hline
\end{tabular}




$$
\begin{array}{ll}
\operatorname{VLR}\left(+45^{\circ}, \pm 90^{\circ}\right)=\left(\begin{array}{ccc}
1 & 0 & 0 \\
0 & 0 & -1 \\
0 & 1 & 0
\end{array}\right)=\mathbf{E}_{3} & \left(\begin{array}{l}
H \\
V \\
S
\end{array}\right) \Longrightarrow\left(\begin{array}{c}
H \\
-S \\
V
\end{array}\right) \\
\operatorname{VLR}\left(-45^{\circ}, \pm 90^{\circ}\right)=\left(\begin{array}{ccc}
1 & 0 & 0 \\
0 & 0 & 1 \\
0 & -1 & 0
\end{array}\right)=\mathbf{E}_{3}^{-1} \quad\left(\begin{array}{c}
H \\
V \\
S
\end{array}\right) \Longrightarrow\left(\begin{array}{c}
H \\
S \\
-V
\end{array}\right)
\end{array}
$$

where the operation $\mathbf{E}_{3}^{-1}$ is also shown in Table 1 .

The entire ensemble of magnets in Fig. 1 can be rolled about the beam axis by $90^{\circ}$. We describe this action by calling this configuration the Horizontal Compact LEP Rotator HLR and note that the operation upon the spin polarization vector is given by the operators $\mathbf{D}_{3}$ and $\mathbf{D}_{3}^{-1}$ in Table $\mathrm{I}$.

\section{Generation of the remaining symmetry operators}

It was shown in $\operatorname{Sec} .2$ how the $\operatorname{VLR}\left( \pm 45^{\circ}, \pm 90^{\circ}\right)$ and $\operatorname{HLR}\left( \pm 90^{\circ}, \pm 45^{\circ}\right)$ configurations are associated with the operators $\mathbf{E}_{3}, \mathbf{E}_{3}^{-1}, \mathbf{D}_{3}$, and $\mathbf{D}_{3}^{-1}$. Reference 1 also describes similar correspondences between the serpents denoted as $\mathbf{V}( \pm \psi, \pm \theta)$ or $\mathbf{H}( \pm \psi, \pm \theta)$ with the 8 operators identified by the letter $\mathbf{A}$ and the two snake operators $\mathbf{S}_{3}$ and $\mathbf{S}_{3}^{-1}$, the Left-Right Snakes $\mathbf{L} \mathbf{R}( \pm \psi, \pm \theta)$ with the operators $\mathbf{D}_{1}$ and $\mathbf{D}_{2}$, and half of an Up-Down snake termed $\mathbf{U D}( \pm \psi, \pm \theta)$ with the corresponding operators $\mathbf{E}_{1}$ and $\mathbf{E}_{2}$. By combining some of these configurations in sequence the remaining operators $\mathbf{C}_{1}, \mathbf{C}_{2}, \mathbf{C}_{3}, \mathbf{S}_{1}$ and $\mathbf{S}_{2}$ can also be generated along with $\mathbf{E}_{3}, \mathbf{E}_{3}^{-1}, \mathbf{D}_{3}$, and $\mathbf{D}_{3}^{-1}$, the four operators which we now find can be obtained more simply without the need of sequential configurations.

A brief summary of these configurations and the corresponding operators cited in Ref. 1 is recalled here:

$$
\begin{array}{rlr}
\mathbf{V}\left( \pm 45^{\circ}, \mp 45^{\circ}\right)=\mathbf{H}\left( \pm 45^{\circ}, \pm 45^{\circ}\right)=\mathbf{S}_{3} & \mathbf{V}\left( \pm 45^{\circ}, \pm 45^{\circ}\right)=\mathbf{H}\left(\mp 45^{\circ}, \pm 45^{\circ}\right)=\mathbf{S}_{3}^{-1} \\
\mathbf{V}\left(+90^{\circ}, \pm 90^{\circ}\right)=\mathbf{A}_{1,2} & \mathbf{V}\left(-90^{\circ}, \pm 90^{\circ}\right)=\mathbf{A}_{3,4} \\
\mathbf{H}\left(+90^{\circ}, \pm 90^{\circ}\right)=\mathbf{A}_{4,3}^{-1} & \mathbf{H}\left(-90^{\circ}, \pm 90^{\circ}\right)=\mathbf{A}_{2,1}^{-1} \\
\mathbf{L R}\left( \pm 90^{\circ},+45^{\circ}\right)=\mathbf{D}_{1} & \mathbf{L R}\left( \pm 90^{\circ},-45^{\circ}\right)=\mathbf{D}_{2} \\
\mathbf{U D}\left(+45^{\circ}, \pm 90^{\circ}\right)=\mathbf{E}_{2} & \mathbf{U D}\left(-45^{\circ}, \pm 90^{\circ}\right)=\mathbf{E}_{1} \\
\mathbf{V L R}\left(+45^{\circ}, \pm 90^{\circ}\right)=\mathbf{E}_{3} & \mathbf{V L R}\left(-45^{\circ}, \pm 90^{\circ}\right)=\mathbf{E}_{3}^{-1} \\
\mathbf{H L R}\left( \pm 90^{\circ},+45^{\circ}\right)=\mathbf{D}_{3} & \mathbf{H L R}\left( \pm 90^{\circ},-45^{\circ}\right)=\mathbf{D}_{3}^{-1}
\end{array}
$$

where the last two lines are the configurations reported here and have now been added. There are 18 operators listed here and we can add the identity operator by setting the fields of all magnets to zero. Next, we note that all 19 operators can be obtained using a single string of 8 magnets ( 4 horizontally bending magnets alternating with 4 vertically 

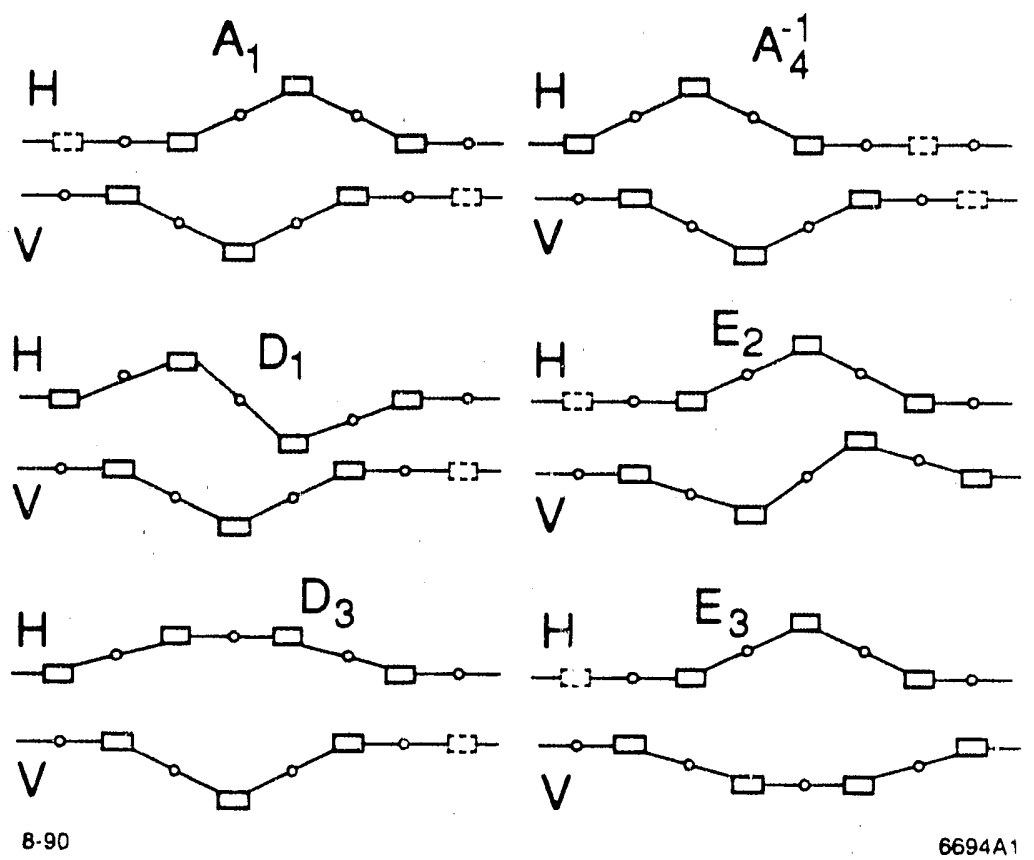

Figure 2: Examples of eight magnets configured to perform different operations.

bending magnets). Examples of how this is done are shown in Fig. 2 and a complete list of operators and corresponding configurations is given in Table II.

\section{Conclusions}

Of the operators shown in Table II, there are 8 which rotate the vertical component of the initial spin polarization vector into the longitudinal direction. These 8 operators are $\mathbf{A}_{1}, \mathbf{A}_{2}, \mathbf{A}_{3}, \mathbf{A}_{4}, \mathbf{E}_{1}, \mathbf{E}_{2}, \mathbf{E}_{3}$, and $\mathbf{E}_{3}^{-1}$. Either examination of Table I or Ref. 1 shows that these 8 are the only operators of the set of 24 which have this property. In Ref. 1, only the first 6 of this list were known to be obtainable from a short simple string of magnets (less than 8 magnets) whereas we now know how to obtain all 8 operators in this manner. By placing a string of 8 magnets on either side of an Interaction Point (IP) it is possible to provide longitudinal polarization at the IP while at the same time generating an overall operation which is desired such as that of a rotator (I), a snake or another of the 24 possibilities. A multiplication table which shows the product of two successive operations whereby the first is one which causes the polarization to be longitudinal can be found in Ref. 1 but is not shown here due to lack of space.

\section{References}

1. T. Fieguth, "Snakes, Rotators, Serpents and the Octahedral Group," Particle Accelerators, 22, No. 3, 183-203 (1987). 
Table II: Configurations using 8 magnets showing the operators generated.

\begin{tabular}{|c|c|c|c|c|c|c|c|c|c|}
\hline Operator & & $\mathrm{Ma}$ & net $\mathrm{Ty}$ & pe and & Precess & on $A n$ & & & Sum of Angles \\
\hline & $\bar{H}$ & 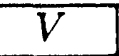 & $H$ & $V$ & $H$ & $\bar{V}$ & $H$ & $V$ & \\
\hline$\overline{A_{1}}$ & - & 90 & 90 & -180 & -180 & 90 & 90 & - & $720^{\circ}$ \\
\hline $\mathbf{A}_{2}$ & - & 90 & -90 & -180 & 180 & 90 & -90 & - & \\
\hline $\mathbf{A}_{3}$ & - & -90 & 90 & 180 & -180 & -90 & 90 & - & \\
\hline $\mathbf{A}_{4}$ & 一 & -90 & -90 & 180 & 180 & -90 & -90 & - & \\
\hline. $\mathbf{A}_{4}^{-1}$ & 90 & 90 & -180 & -180 & 90 & 90 & - & - & - \\
\hline $\mathbf{A}_{3}^{-1}$ & -90 & 90 & 180 & -180 & -90 & 90 & - & - & \\
\hline$A_{2}^{-1}$ & 90 & -90 & -180 & 180 & 90 & -90 & - & - & \\
\hline $\mathbf{A}_{1}^{-1}$ & -90 & -90 & 180 & 180 & -90 & -90 & - & - & \\
\hline $\mathrm{D}_{1}$ & 45 & 90 & -90 & -180 & 90 & 90 & -45 & - & $630^{\circ}$ \\
\hline & 45 & -90 & -90 & 180 & 90 & -90 & -45 & - & \\
\hline $\mathrm{D}_{2}$ & -45 & 90 & 90 & -180 & -90 & 90 & 45 & - & \\
\hline & -45 & -90 & 90 & 180 & -90 & -90 & 45 & - & \\
\hline $\mathbf{E}_{1}$ & - & -45 & 90 & 90 & -180 & -90 & 90 & 45 & \\
\hline & - & -45 & -90 & 90 & 180 & -90 & -90 & 45 & \\
\hline $\mathbf{E}_{2}$ & - & 45 & 90 & -90 & -180 & .90 & 90 & -45 & \\
\hline & - & 45 & -90 & -90 & 180 & 90 & -90 & -45 & \\
\hline$\overline{\mathbf{D}_{3}}$ & 45 & 90 & -45 & -180 & -45 & 90 & 45 & - & $540^{\circ}$ \\
\hline & 45 & -90 & -45 & 180 & -45 & -90 & 45 & - & \\
\hline $\mathrm{D}_{3}^{-1}$ & -45 & 90 & 45 & -180 & 45 & 90 & -45 & - & \\
\hline & -45 & -90 & 45 & 180 & 45 & -90 & -45 & - & \\
\hline $\mathbf{E}_{3}$ & - & 45 & 90 & -45 & -180 & -45 & 90 & 45 & \\
\hline & - & 45 & -90 & -45 & 180 & -45 & -90 & 45 & \\
\hline $\mathbf{E}_{3}^{-1}$ & - & -45 & 90 & 45 & -180 & 45 & 90 & -45 & \\
\hline & - & -45 & -90 & 45 & 180 & 45 & -90 & -45 & \\
\hline $\mathbf{S}_{3}$ & 45 & 45 & -90 & -90 & 45 & 45 & - & - & $360^{\circ}$ \\
\hline & -45 & -45 & 90 & 90 & -45 & -45 & - & - & \\
\hline & & 45 & -45 & -90 & 90 & 45 & -45 & - & \\
\hline & & -45 & 45 & 90 & -90 & -45 & 45 & - & \\
\hline $\mathrm{S}_{3}^{-1}$ & & 45 & 45 & -90 & -90 & 45 & 45 & - & \\
\hline & & -45 & -45 & 90 & 90 & -45 & -45 & - & \\
\hline & 45 & -45 & -90 & 90 & 45 & -45 & - & - & \\
\hline & -45 & 45 & 90 & -90 & -45 & 45 & - & - & \\
\hline $\begin{array}{c}\mathrm{BL} \max \\
(\mathrm{T}-\mathrm{m})\end{array}$ & 2.309 & 2.309 & 4.618 & 4.618 & 4.618 & 2.309 & 2.309 & 1.155 & \\
\hline
\end{tabular}

2. G. Guignard and J. P. Gourber, "Feasibility Study of Compact and Tunable Spin Rotators," CERN SL/90-04 (AP).

3. B. W. Montague, "Polarized Beams in High Energy Storage Rings," Physics Reports, 113, No. 1, 8-13 (1984). 


\section{DISCLAIMER}

This report was prepared as an account of work sponsored by an agency of the United States Government. Neither the United States Government nor any agency thereof, nor any of their employees, makes any warranty, express or implied, or assumes any legal liability or responsibility for the accuracy, completeness, or usefulness of any information, apparatus, product, or process disclosed, or represents that its use would not infringe privately owned rights. Reference herein to any specific commercial product, process, or service by trade name, trademark, manufacturer, or otherwise does not necessarily constitute or imply its endorsement, recommendation, or favoring by the United States Government or any agency thereof. The views and opinions of authors expressed herein do not necessarily state or reflect those of the United States Government or any agency thereof. 

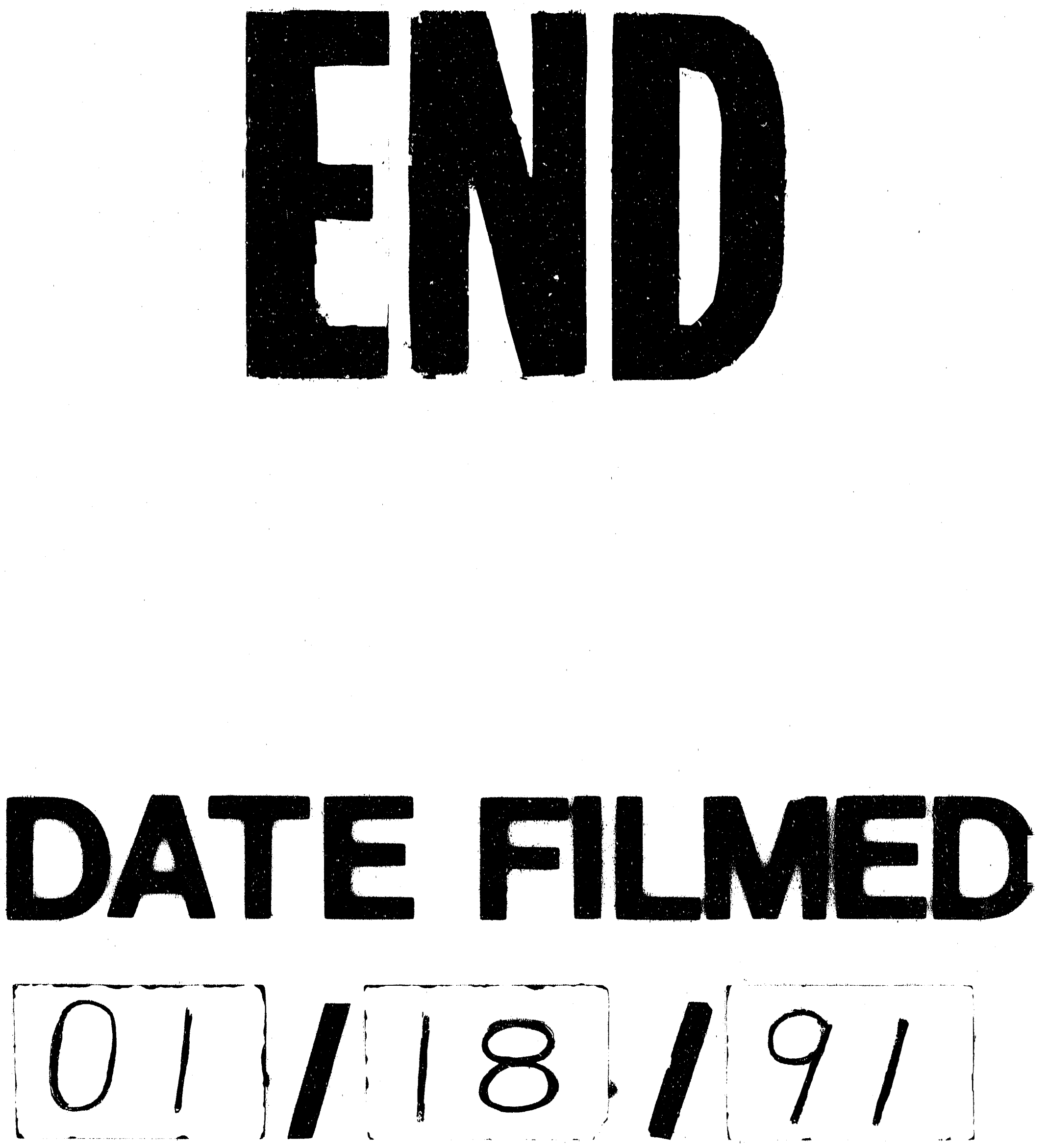
